\title{
Ischemia in three left ventricular regions: Insights into the pathogenesis of acute ischemic mitral regurgitation
}

\author{
Tomasz A. Timek, MD $D^{a, b}$ \\ David T. Lai, FRACS \\ Frederick Tibayan, $\mathrm{MD}^{\mathrm{a}}$ \\ David Liang, MD, PhD \\ George T. Daughters, MS ${ }^{a, d}$ \\ Paul Dagum, MD, $\mathrm{PhD}^{\mathrm{a}}$ \\ Mary K. Zasio, $\mathrm{BA}^{\mathrm{a}}$ \\ Sidney Lo, MD \\ Trevor Hastie, $\mathrm{PhD}^{\mathrm{e}}$ \\ Neil B. Ingels, Jr, PhD ${ }^{\mathrm{a}, \mathrm{d}}$ \\ D. Craig Miller, $M D^{a}$
}

\begin{abstract}
From the Department of Cardiovascular and Thoracic Surgery, ${ }^{\mathrm{a}}$ Division of Cardiovascular Medicine, ${ }^{\mathrm{c}}$ and the Department of Biostatistics, ${ }^{\text {e }}$ Stanford University School of Medicine, Stanford; the Department of Surgery, ${ }^{\mathrm{b}}$ Loma Linda University Medical Center, Loma Linda; and the Laboratory of Cardiovascular Physiology and Biophysics, ${ }^{\text {d }}$ Research Institute of the Palo Alto Medical Foundation, Palo Alto, Calif

Supported by grants HL-29589 and HL67025 from the National Heart, Lung, and Blood Institute. Drs Timek, Tibayan, Dagum, and Lai are Carl and Leah McConnell Cardiovascular Surgical Research Fellows. Drs Timek, Dagum, and Tibayan were also supported by NHLBI Individual Research Service Awards HL-10452, HL-10000, and HL-67563, respectively. Dr Timek is also a recipient of the Thoracic Surgery Foundation Research Fellowship Award. Dr Lai was supported by a fellowship from the American Heart Association, Western States Affiliate.
\end{abstract}

Read at the Eighty-second Annual Meeting of The American Association for Thoracic Surgery, Washington, DC, May 5-8, 2002.

Received for publication April 29, 2002; revisions requested July 8 , 2002; revisions received Aug 4, 2002; accepted for publication Aug 15, 2002.

Address for reprints: D. Craig Miller, MD, Department of Cardiothoracic Surgery, Falk Cardiovascular Research Center, Stanford University School of Medicine, Stanford, CA 94305-5247 (E-mail: dcm@stanford.edu).

J Thorac Cardiovasc Surg 2003;125:559-69

Copyright ( 92003 by The American Association for Thoracic Surgery

$0022-5223 / 2003 \$ 30.00+0$

doi: $10.1067 / \mathrm{mtc} .2003 .43$
Background: Acute posterolateral left ventricular ischemia in sheep results in ischemic mitral regurgitation, but the effects of ischemia in other left ventricular regions on ischemic mitral regurgitation is unknown.

Methods: Six adult sheep had radiopaque markers placed on the left ventricle, mitral annulus, and anterior and posterior mitral leaflets at the valve center and near the anterior and posterior commissures. After 6 to 8 days, animals were studied with biplane videofluoroscopy and transesophageal echocardiography before and during sequential balloon occlusion of the left anterior descending, distal left circumflex, and proximal left circumflex coronary arteries. Time of valve closure was defined as the time when the distance between leaflet edge markers reached its minimum plateau, and systolic leaflet edge separation distance was calculated on the basis of left ventricular ejection.

Results: Only proximal left circumflex coronary artery occlusion resulted in ischemic mitral regurgitation, which was central and holosystolic. Delayed valve closure (anterior commissure, $58 \pm 29$ vs $92 \pm 24 \mathrm{~ms}$; valve center, $52 \pm 26$ vs $92 \pm 23 \mathrm{~ms}$; posterior commissure, $60 \pm 30 \mathrm{vs} 94 \pm 14 \mathrm{~ms}$; all $P<.05)$ and increased leaflet edge separation distance during ejection (mean increase, $2.2 \pm$ $1.5 \mathrm{~mm}, 2.1 \pm 1.9 \mathrm{~mm}$, and $2.1 \pm 1.5 \mathrm{~mm}$ at the anterior commissure, valve center, and posterior commissure, respectively; $P<.05$ for all) was seen during proximal left circumflex coronary artery occlusion but not during left anterior descending or distal left circumflex coronary artery occlusion. Ischemic mitral regurgitation was associated with a $19 \% \pm 10 \%$ increase in mitral annular area, and displacement of both papillary muscle tips away from the septal annulus at end systole.

Conclusions: Acute ischemic mitral regurgitation in sheep occurred only after proximal left circumflex coronary artery occlusion along with delayed valve closure in early systole and increased leaflet edge separation throughout ejection in all 3 leaflet coaptation sites. The degree of left ventricular systolic dysfunction induced did not correlate with ischemic mitral regurgitation, but both altered valvular and subvalvular 3-dimensional geometry were necessary to produce ischemic mitral regurgitation during acute left ventricular ischemia. 
I schemic mitral regurgitation (IMR) presents a challenging clinical problem associated with suboptimal surgical results and poor prognosis caused by the underlying left ventricular (LV) dysfunction caused by myocardial ischemia and infarction..$^{1-3}$ Mitral valve repair might offer better outcomes in this patient population than valve replacement, ${ }^{4}$ but no standardized repair methods exist on the basis of the various leaflet-motion abnormalities seen. Despite a plethora of experimental and clinical studies investigating the 3-dimensional (3-D) valvular and subvalvular geometric perturbations associated with IMR, knowledge of the precise mechanisms responsible for valvular incompetence remains elusive. Mitral annular changes, ${ }^{5,6}$ systolic leaflet apical tethering, ${ }^{6-10}$ more spherical LV shape, ${ }^{11,12}$ and impaired LV systolic function ${ }^{13}$ have all been suggested as contributing factors, but a more detailed understanding of the underlying mechanisms is needed to guide better surgical treatment.

In prior studies with radiopaque marker methods in sheep, we showed that proximal left circumflex (LCx) coronary artery occlusion results in acute posterolateral LV ischemia and IMR caused by delayed valve closure during very early systole ${ }^{5}$ and perturbed leaflet $3-\mathrm{D}$ geometry throughout ejection. ${ }^{14}$ Others have attributed acute IMR in similar animal models to papillary muscle discoordination at end systole. ${ }^{10}$ These and related studies had limitations, one of which was that neither the timing nor the location of the regurgitant jet could be determined from transthoracic $^{5,14}$ or subdiaphragmatic ${ }^{10}$ echocardiography. Therefore the observed 3-D perturbations in valvular and subvalvular dynamic geometry arising during acute ischemia could not be correlated either spatially or temporally with the echocardiographic regurgitant leak nor could the pathogenesis of IMR as a function of ischemia in other LV regions be examined. Our previous experiments examined leaflet dynamics only at the central edge of the leaflets not allowing detection of possible changes occurring near the commissures; also, the effects of ischemia in other LV regions on mitral competence and valvular dynamics was not examined. To gain more insight into the mechanistic pathogenesis of IMR, we used transesophageal Doppler echocardiography and biplane videofluoroscopy of multiple leaflet markers to study the effects of posterolateral LV ischemia caused by proximal LCx (pLCx) coronary artery occlusion and acute ischemia in other LV regions (ie, occlusion of the left anterior descending [LAD] coronary artery or distal $\mathrm{LCx}[\mathrm{dLCx}]$ coronary artery) on mitral valve $3-\mathrm{D}$ dynamic geometry and competence.

\section{Methods}

\section{Surgical Preparation}

The surgical preparation has previously been described ${ }^{15}$ and will be presented only in abbreviated form. Six adult sheep $(71 \pm 13$ $\mathrm{kg}$ ) were premedicated with ketamine $(25 \mathrm{mg} / \mathrm{kg}$ administered intramuscularly), and anesthesia was induced with sodium thiopental $(6.8 \mathrm{mg} / \mathrm{kg}$ administered intravenously) and maintained with inhalational isoflurane (1\%-2.5\%). Through a left thoracotomy, 8 tantalum myocardial markers (inner diameter, $0.8 \mathrm{~mm}$; outer diameter, $1.3 \mathrm{~mm}$; length, $1.5-3.0 \mathrm{~mm}$ ) were inserted in the LV epicardial layer along 4 equally spaced longitudinal meridians, as shown in Figure 1. After establishment of cardiopulmonary bypass with the heart arrested, a single tantalum marker was placed at the tip and base of both papillary muscles (Figure 1), and 8 tantalum markers were sutured around the circumference of the mitral annulus (one near each commissure and 3 along the septal and lateral annulus, Figure 2). Two miniature gold markers were sutured at the central edge of each leaflet, and 4 additional markers were sewn on each leaflet edge halfway between the central leaflet edge marker and the anterior and posterior commissures (Figure 2). After completion of marker placement, the animal was rewarmed, the atriotomy was closed, the crossclamp was removed, the heart was resuscitated, and the animal was weaned from bypass. Cardiopulmonary bypass time was $81 \pm 6$ minutes, with an aortic crossclamp time of $61 \pm 3$ minutes. A micromanometer pressure transducer (PA4.5-X6, Konigsberg Instruments, Inc) was placed in the LV chamber through the apex. Proper myocardial marker position was confirmed in all animals on postmortem examination.

\section{Experimental Protocol}

After $7 \pm 1$ (mean $\pm 1 \mathrm{SD}$ ) days, each animal was taken to the cardiac catheterization laboratory, sedated with ketamine (1-4 mg $\times \mathrm{kg}^{-1} \times \mathrm{h}^{-1}$ administered intravenously) and diazepam (5 mg intravenous bolus as needed), intubated, and mechanically ventilated (veterinary anesthesia ventilator 2000, Halowell EMC). An esmolol intravenous infusion (20-50 $\left.\mu \mathrm{g} \times \mathrm{kg}^{-1} \times \mathrm{min}^{-1}\right)$ minimized reflex sympathetic responses. Coronary artery balloon occlusion (1-4 minutes), as described previously, ${ }^{5}$ was performed at 3 locations in sequential fashion: proximal LAD coronary artery (proximal to the first diagonal), dLCx coronary artery (distal to the second obtuse marginal), and pLCx coronary artery (proximal to the first obtuse marginal).

Videofluoroscopic marker image recordings were acquired before and during induction of ischemia at each location with simultaneous transesophageal color Doppler echocardiography to assess mitral regurgitation (MR). After each data-acquisition run, a 3- to 5 -minute period of stabilization was allowed before data acquisitions were repeated. MR was graded on the basis of regurgitant jet extent and width as none $(0)$, mild $(+1)$, moderate $(+2)$, moderate to severe $(+3)$, or severe $(+4)$ by an experienced echocardiographer (D.L.).

All animals received humane care in compliance with the "Principles of Laboratory Animal Care" formulated by the National Society for Medical Research and the "Guide for Care and Use of Laboratory Animals" prepared by the National Academy of Sciences and published by the National Institutes of Health (DHEW NIHG publication no. 85-23, revised 1985). This study was approved by the Stanford University Medical School Laboratory Research Animal Review committee and conducted according to Stanford University policy. 


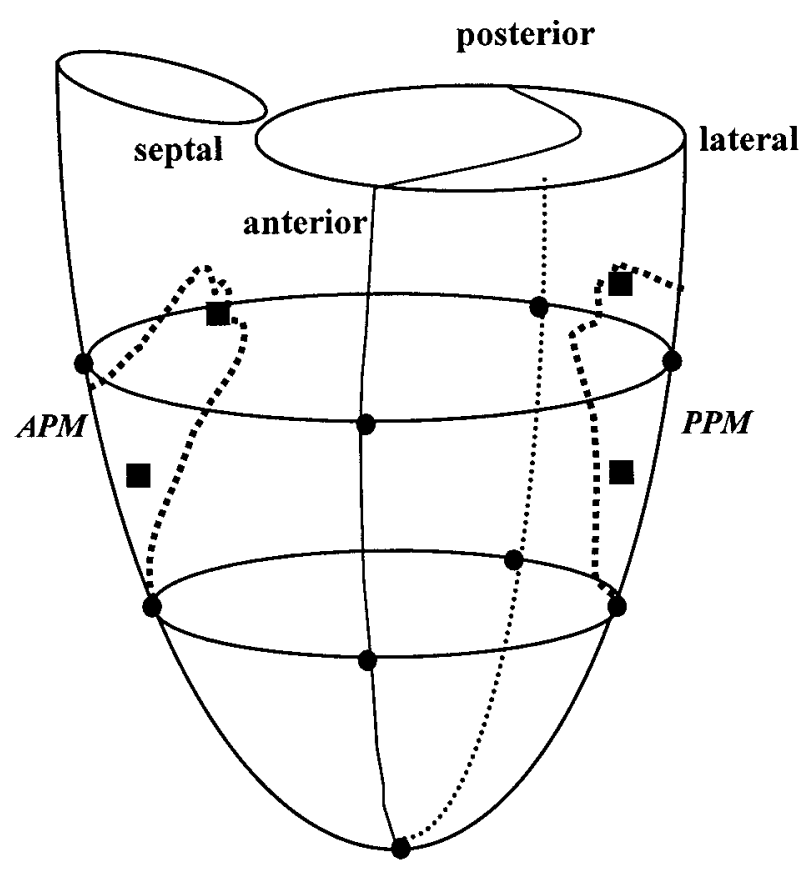

Figure 1. Miniature radiopaque marker array (filled circles, myocardial; filled squares, papillary) used for the left ventricle in this experimental study. PPM, Posterior papillary muscle; APM, anterior papillary muscle.

\section{Data Acquisition}

Images were acquired with the animal in the right lateral decubitus position with a Philips Optimus 2000 biplane Lateral ARC 2/Poly DIAGNOST C2 system (Philips Medical Systems), with the image intensifier in the 9-inch fluoroscopic mode. Data from 2 radiographic views were digitized and merged to yield 3-D coordinates for each of the radiopaque markers every $16.7 \mathrm{~ms}$ by using custom-designed software. ${ }^{16}$ Ascending aortic pressure, LV pressure, and electrocardiographic voltage signals were digitized and recorded simultaneously during marker data acquisition.

\section{Data Analysis}

Hemodynamics and cardiac cycle timing markers. Two to 3 consecutive steady-state beats immediately before occlusion of any coronary artery were averaged and defined as preischemia data for each animal. Similarly, 2 to 3 beats during occlusion of the LAD, dLCx, and pLCx coronary arteries were averaged and defined as ischemia data. During each cardiac cycle, end systole was defined as the time of the videofluoroscopic frame containing the point of peak negative rate of $\mathrm{LV}$ pressure decrease $(-\mathrm{dP} / \mathrm{dt})$, whereas end diastole was defined as the videofluoroscopic frame containing the peak of the electrocardiographic $\mathrm{R}$ wave. Instantaneous LV volume was calculated from the positions of the epicardial LV markers and annular markers by using a space-filling multiple tetrahedral volume method for each frame (ie, every 16.7 ms). ${ }^{17}$ Although myocardial volume is included in calculation of LV volume, relative changes in LV chamber size are accurately measured by using the epicardial marker coordinates.

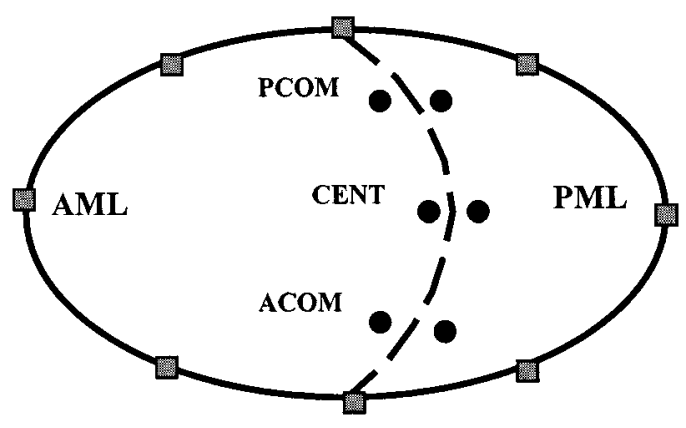

Figure 2. Schematic diagram of the implanted mitral annular (filled squares) and mitral leaflet (filled circles) markers. Leaflet edge marker pairs were implanted at the valve center (CENT), halfway between the center and the anterior commissure (ACOM), and halfway between the center and the posterior commissure (PCOM). AML, Anterior mitral leaflet; $P M L$, posterior mitral leaflet.

Mitral leaflet and annular dynamics. Mitral valve-closure time was defined as the time at which leaflet edge separation reached its minimum plateau. Leaflet separation was measured as distance in 3-D space between the 2 leaflet edge markers at the center of the valve and toward the anterior and posterior commissures (Figure 2). Leaflet edge separation throughout the cardiac cycle for each animal was represented by a unique continuous function generated by spline-fitting computer software (S-Plus, Mathsoft, Inc) to identify objectively the time of valve closure. Valve closure for each leaflet region (anterior commissure, valve center, and posterior commissure) was defined as the time after end diastole at which the first time derivative with respect to time of the associated spline function decreased to less than $0.002 \mathrm{~cm} / \mathrm{s}$ (a value reflecting the beginning of the leaflet edge distance minimum plateau). Leaflet edge separation at each leaflet edge marker pair was determined for each experimental condition at $25 \%, 50 \%$, $75 \%$, and $100 \%$ of LV ejected LV stroke volume to assess leaflet separation during systole. Mitral annular area was calculated as the sum of the areas of 8 triangles formed by consecutive adjacent marker pairs on the annulus and the annular centroid. The septallateral (S-L) diameter of the annulus (clinically referred to as the anterior-posterior dimension) was calculated as the distance in 3-D space between the 2 markers placed in the middle of the septal and lateral mitral annulus, respectively. The commissure-commissure (C-C) diameter was calculated as the distance between the 2 annular commissural markers. Papillary muscle percentage shortening was determined as the change from end diastole to end systole in distance between the papillary muscle tip and base markers divided by the distance at end diastole.

Statistical analysis. All data are reported as means \pm 1 SD. Hemodynamic and marker-derived data from 2 to 3 consecutive steady-state beats that were time aligned at end diastole and data from these beats were averaged for each animal. The data were analyzed over 20 frames before and 20 frames after end diastole (total time of $667 \mathrm{~ms}$ ), thereby allowing assessment of variables over the entire cardiac cycle. Data were compared by using the Student $t$ test for paired comparisons and repeated-measures analysis of variance (ANOVA) when appropriate. 
TABLE 1. Hemodynamics

\begin{tabular}{|c|c|c|c|c|c|c|}
\hline & \multicolumn{2}{|c|}{ LAD } & \multicolumn{2}{|c|}{ dLCx } & \multicolumn{2}{|c|}{ pLCx } \\
\hline & Before ischemia & Ischemia & Before ischemia & Ischemia & Before ischemia & Ischemia \\
\hline $\mathrm{HR}\left(\min ^{-1}\right)$ & $96 \pm 14$ & $100 \pm 16$ & $98 \pm 17$ & $92 \pm 25$ & $103 \pm 14$ & $83 \pm 20$ \\
\hline $\mathrm{LV} \mathrm{dP/dt} \mathrm{max}_{\max }(\mathrm{mm} / \mathrm{s})$ & $1453 \pm 512$ & $993 \pm 377^{*}$ & $1730 \pm 545$ & $1192 \pm 417^{*}$ & $1596 \pm 545$ & $979 \pm 300$ \\
\hline $\mathrm{EDV}(\mathrm{mL})$ & $155 \pm 40$ & $162 \pm 38$ & $155 \pm 35$ & $162 \pm 42$ & $156 \pm 37$ & $166 \pm 41^{*}$ \\
\hline ESV (mL) & $118 \pm 37$ & $137 \pm 42^{*}$ & $114 \pm 28$ & $129 \pm 35^{*}$ & $117 \pm 31$ & $138 \pm 33^{*}$ \\
\hline$S V(m L)$ & $37 \pm 10$ & $27 \pm 6^{*}$ & $42 \pm 12$ & $32 \pm 9^{*}$ & $39 \pm 9$ & $29 \pm 9^{*}$ \\
\hline LVESP (mm Hg) & $60 \pm 6$ & $47 \pm 11^{*}$ & $64 \pm 11$ & $55 \pm 16$ & $75 \pm 9$ & $47 \pm 16^{*}$ \\
\hline $\mathrm{LVP}_{\text {max }}(\mathrm{mm} \mathrm{Hg})$ & $103 \pm 11$ & $74 \pm 14^{*}$ & $114 \pm 10$ & $85 \pm 21^{*}$ & $116 \pm 13$ & $73 \pm 21^{*}$ \\
\hline
\end{tabular}

$H R$, Heart rate; $d P / d t_{\text {max }}$ maximum positive rate of change of LV pressure; $E D V$, LV end-diastolic volume; $E S V$, LV end-systolic volume; $S V$, stroke volume; LVESP, end-systolic LV pressure; $L V P_{\text {max }}$ maximum LV pressure.

${ }^{*} P<.05$ versus before ischemia.

TABLE 2. Valve-closure times

\begin{tabular}{lccc}
\hline & \multicolumn{3}{c}{ Valve-closure time (ms) } \\
\cline { 2 - 3 } & Before ischemia & Ischemia & \\
\hline LAD & & & \\
ACOM & $61 \pm 19$ & $66 \pm 22$ & .64 \\
CENT & $56 \pm 18$ & $72 \pm 23$ & .35 \\
PCOM & $67 \pm 31$ & $73 \pm 31$ & .73 \\
dLCX & $51 \pm 16$ & $59 \pm 42$ & .12 \\
ACOM & $47 \pm 20$ & $63 \pm 34$ & .32 \\
CENT & $54 \pm 28$ & $92 \pm 24$ & .49 \\
PCOM & $58 \pm 29$ & $92 \pm 23$ & .049 \\
pLCX & $52 \pm 26$ & $94 \pm 14$ & .025 \\
ACOM & $60 \pm 30$ & .028 \\
CENT & & &. \\
PCOM & & & \\
\hline
\end{tabular}

$\angle A D$, LAD coronary artery occlusion; $A C O M$, leaflet edge marker pair near the anterior commissure; $C E N T$, leaflet edge marker pair at valve center; $P C O M$, leaflet edge marker pair near the posterior commissure; $d L C x, \mathrm{dLCx}$ coronary artery occlusion; $p L C x$, $\mathrm{pLCx}$ coronary artery occlusion.

\section{Results}

Table 1 summarizes the hemodynamic variables during acute occlusion of the LAD, $\mathrm{dLCx}$, and $\mathrm{pLCx}$ coronary arteries relative to the corresponding preischemic control conditions. $\mathrm{LV} \mathrm{dP} / \mathrm{dt}$ decreased a similar amount during ischemia in the 3 different $\mathrm{LV}$ regions $(32 \% \pm 7 \%, 32 \% \pm$ $12 \%$, and $36 \% \pm 24 \%$ for LAD, dLCx, and pLCx coronary arteries, respectively; $P=.88$, ANOVA), as did peak LV pressure $(28 \% \pm 12 \%, 26 \% \pm 14 \%$, and $37 \% \pm 15 \%$ for $\mathrm{LAD}, \mathrm{dLCx}$, and $\mathrm{pLCx}$ coronary arteries, respectively; $P=$ .19, ANOVA) and stroke volume $(22 \% \pm 11 \%, 21 \% \pm 9 \%$, and $26 \% \pm 13 \%$ for $\mathrm{LAD}, \mathrm{dLCx}$, and pLCx coronary arteries, respectively; $P=.52$, ANOVA). Heart rate, LV $\mathrm{dP} / \mathrm{dt}, \mathrm{LV}$ end-diastolic and end-systolic volumes, LV enddiastolic and end-systolic pressures, stroke volume, and peak LV pressure during each arterial occlusion did not differ from those seen in the other 2 ischemic interventions $(P>.05$, ANOVA). The difference in end-diastolic volume among the 3 interventions approached significance $(P=$ .056 , ANOVA); a paired $t$ test with Bonferroni correction revealed that end-diastolic volume was significantly differ- ent only between the dLCX and pLCX coronary artery occlusions, and the increase in LV end-diastolic volume during ischemia was significant only for pLCX coronary artery occlusion. Thus LV systolic function was decreased by approximately the same extent during the 3 different ischemic insults. Heart rate did not change.

\section{Mitral Regurgitation}

The amount of MR present during baseline conditions before each ischemic insult did not change throughout the experiment. The average baseline MR grade was $+0.5 \pm$ 0.5 , with 2 animals having no MR, 2 having trace MR, and 2 having mild MR. During LAD and dLCx coronary artery occlusion, MR increased slightly to $+0.7 \pm 0.8$ and $+0.8 \pm$ 1.1, respectively $(P>.2$ for both vs preischemic control). During acute $\mathrm{pLCx}$ coronary artery occlusion, however, MR increased significantly from $+0.5 \pm 0.5$ to $+2.0 \pm 1.1(P=$ .007), with one animal having severe regurgitation, 2 having moderate regurgitation, 2 having mild-to-moderate regurgitation, and 1 having mild regurgitation. The origin of the MR jet by means of color Doppler echocardiography was 

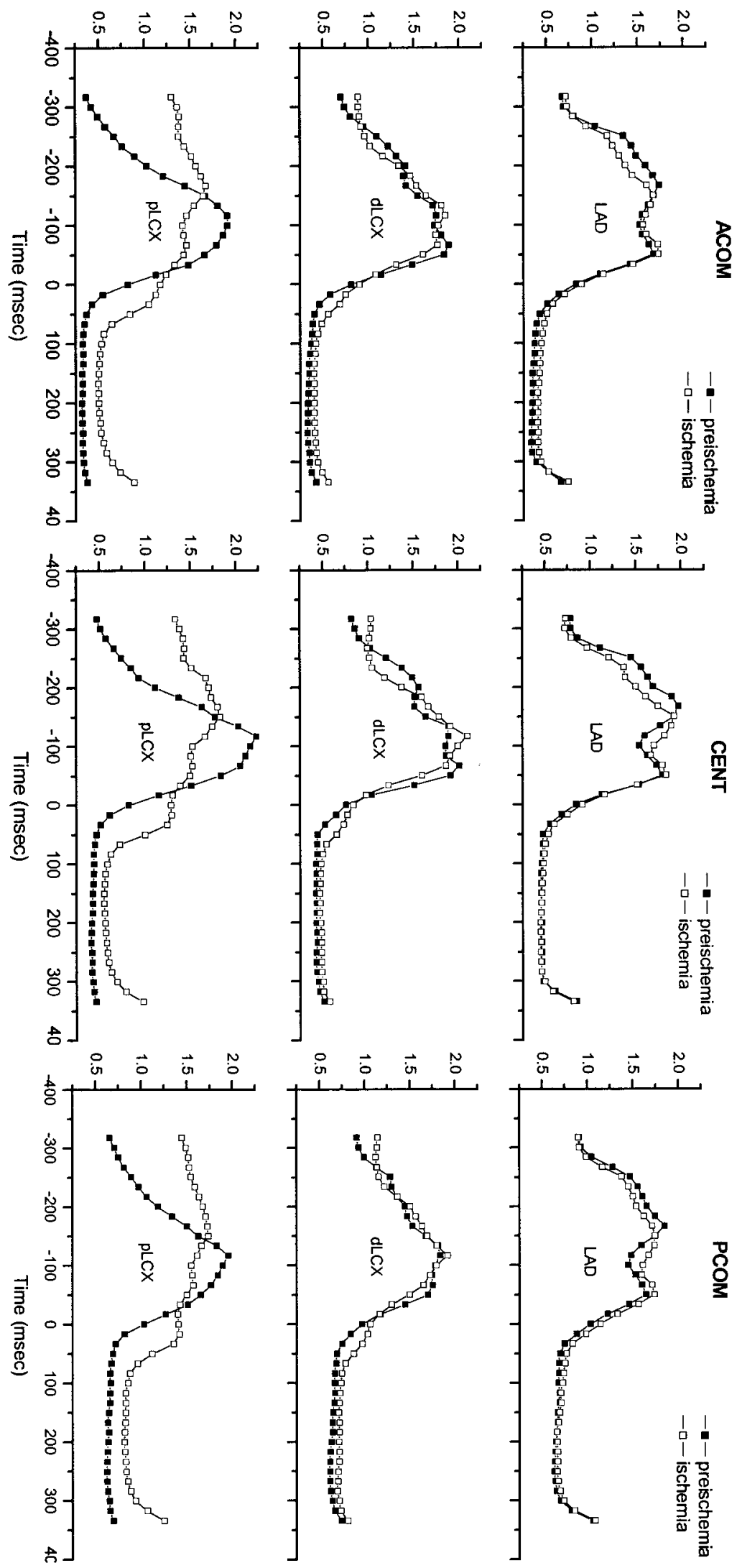

Figure 3. Leaflet edge separation for all animals before (filled squares) and during (open circles) acute LV ischemia induced by balloon occlusion of either the LAD coronary artery (top row), the dLCx coronary artery (middle row), or the $\mathrm{pLCx}$ coronary artery (bottom row). Leaflet edge separation was measured near the anterior commissure (ACOM, left column), at the valve center (CENT, middle column), and near the posterior commissure (PCOM, right column). A 650-ms time interval centered at end diastole $(t=0)$ is shown for all 3 groups. 
TABLE 3. Leaflet edge separation during ejection

\begin{tabular}{|c|c|c|c|c|c|c|c|c|}
\hline & \multicolumn{8}{|c|}{ Leaflet edge separation $(\mathrm{mm})$ at $\%$ LV ejection } \\
\hline & \multicolumn{4}{|c|}{ Before ischemia } & \multicolumn{4}{|c|}{ Ischemia } \\
\hline & $25 \%$ & $50 \%$ & $75 \%$ & $100 \%$ & $25 \%$ & $50 \%$ & $75 \%$ & $100 \%$ \\
\hline \multicolumn{9}{|l|}{ LAD } \\
\hline ACOM & $3.7 \pm 1.2$ & $3.5 \pm 1.0$ & $3.5 \pm 0.9$ & $3.7 \pm 1.1$ & $4.6 \pm 1.9$ & $4.3 \pm 1.6$ & $4.1 \pm 1.4$ & $4.1 \pm 1.4$ \\
\hline CENT & $4.7 \pm 1.9$ & $4.6 \pm 1.7$ & $4.5 \pm 1.6$ & $4.7 \pm 1.8$ & $4.9 \pm 2.1$ & $4.7 \pm 1.9$ & $4.5 \pm 1.9$ & $4.5 \pm 1.5$ \\
\hline $\begin{array}{l}\text { PCOM } \\
\mathrm{dLCx}\end{array}$ & $6.9 \pm 2.5$ & $6.7 \pm 2.6$ & $6.5 \pm 2.6$ & $6.8 \pm 3.0$ & $7.4 \pm 2.9$ & $7.0 \pm 2.9$ & $6.7 \pm 2.9$ & $6.9 \pm 2.9$ \\
\hline ACOM & $3.6 \pm 1.1$ & $3.5 \pm 1.1$ & $3.3 \pm 1.0$ & $3.4 \pm 1.1$ & $4.2 \pm 1.6^{*}$ & $4.0 \pm 1.6$ & $4.0 \pm 1.7$ & $4.2 \pm 1.8$ \\
\hline CENT & $4.3 \pm 1.9$ & $4.3 \pm 1.8$ & $4.3 \pm 1.8$ & $4.1 \pm 1.2$ & $4.7 \pm 2.0$ & $4.6 \pm 1.9$ & $5.1 \pm 1.7$ & $4.9 \pm 1.4$ \\
\hline $\begin{array}{l}\text { PCOM } \\
\text { pLCx }\end{array}$ & $6.6 \pm 2.8$ & $6.5 \pm 2.7$ & $6.3 \pm 2.8$ & $6.2 \pm 2.8$ & $7.2 \pm 2.9$ & $7.2 \pm 2.8$ & $7.1 \pm 2.8$ & $7.0 \pm 2.8^{*}$ \\
\hline ACOM & $3.2 \pm 0.8$ & $3.3 \pm 0.7$ & $3.3 \pm 0.7$ & $3.4 \pm 0.7$ & $6.2 \pm 1.8^{*}$ & $5.2 \pm 1.4^{*}$ & $4.9 \pm 1.5^{*}$ & $5.7 \pm 2.0^{*}$ \\
\hline CENT & $4.0 \pm 1.9$ & $4.3 \pm 1.8$ & $4.1 \pm 1.9$ & $4.3 \pm 1.8$ & $6.8 \pm 1.8^{*}$ & $5.6 \pm 1.7^{*}$ & $5.5 \pm 1.6^{*}$ & $7.4 \pm 2.3^{*}$ \\
\hline РСOM & $6.6 \pm 2.7$ & $6.4 \pm 3.0$ & $6.3 \pm 3.0$ & $6.4 \pm 3.0$ & $8.9 \pm 2.7^{*}$ & $8.3 \pm 2.9^{*}$ & $8.2 \pm 3.0^{*}$ & $8.5 \pm 3.0^{*}$ \\
\hline
\end{tabular}

$\angle A D$, LAD coronary artery occlusion; $A C O M$, leaflet edge marker pair near the anterior commissure; CENT, leaflet edge marker pair at valve center; $P C O M$, leaflet edge marker pair near the posterior commissure; $d L C x, \mathrm{dLCx}$ coronary artery occlusion; $p L C x, \mathrm{pLC}$ coronary artery occlusion.

${ }^{*} P<.05$ versus before ischemia.

TABLE 4. Mitral annular dynamics during LAD, pLCx, and dLCx occlusion

\begin{tabular}{|c|c|c|c|c|c|c|}
\hline & \multicolumn{2}{|c|}{ LAD } & \multicolumn{2}{|c|}{ dLCx } & \multicolumn{2}{|c|}{$\mathrm{pLCx}$} \\
\hline & Before ischemia & Ischemia & Before ischemia & Ischemia & Before ischemia & Ischemia \\
\hline $\mathrm{MAA}_{\mathrm{ED}}\left(\mathrm{cm}^{2}\right)$ & $7.39 \pm 2.02$ & $7.59 \pm 1.44$ & $7.25 \pm 1.71$ & $7.76 \pm 1.78^{*}$ & $7.11 \pm 1.51$ & $8.46 \pm 2.01^{*}$ \\
\hline$S L_{E D}(\mathrm{~cm})$ & $2.64 \pm .32$ & $2.77 \pm .20$ & $2.61 \pm .24$ & $2.79 \pm .25^{*}$ & $2.57 \pm .20$ & $3.02 \pm .30^{*}$ \\
\hline $\mathrm{CC}_{\mathrm{ED}}(\mathrm{cm})$ & $3.64 \pm .52$ & $3.68 \pm .44$ & $3.66 \pm .47$ & $3.67 \pm .47$ & $3.63 \pm .44$ & $3.75 \pm .50$ \\
\hline
\end{tabular}

$L A D$, LAD coronary artery occlusion; $d L C x, \mathrm{dLCx}$ coronary artery occlusion; $p L C x, \mathrm{pLCx}$ coronary artery occlusion; $M A A_{E D}$, End-diastolic mitral annular area; $S L_{E D}$, end-diastolic mitral annular septal-lateral diameter; $C C_{E D}$, end-diastolic mitral annular commissure-commissure diameter.

${ }^{*} P<.05$ versus before ischemia.

central in 5 of the 6 animals and posterolateral in 1 of the animals. Evaluation of the timing of the regurgitant jet was feasible in 4 animals: all 4 had holosystolic MR.

\section{Mitral Valve Closure and Leaflet Edge Separation}

Valve-closure times with respect to end diastole for each ischemic intervention and at each leaflet edge marker pair location (anterior commissure, valve center, and posterior commissure) are summarized in Table 2. Neither LAD nor $\mathrm{dLCx}$ coronary artery occlusion altered valve-closure time, but $\mathrm{pLCx}$ coronary artery occlusion significantly delayed valve closure at all 3 leaflet locations. Leaflet edge separation throughout the cardiac cycle for each leaflet edge marker pair during the 3 ischemic interventions is shown in Figure 3. Delayed early systolic valve closure (leaflet loitering) was observed during pLCx coronary artery occlusion but not during LAD or dLCx coronary artery occlusion. Leaflet edge separation was greater during systole versus preischemic control conditions during $\mathrm{pLCx}$ coronary artery occlusion, but this was not observed during the other 2 ischemic interventions (Figure 3). Leaflet edge separation distance was calculated at $25 \%, 50 \%, 75 \%$, and $100 \%$ of LV ejection (Table 3) and was larger at all times and in all 3 coaptation regions during pLCx coronary artery occlusion compared with that seen during preischemic control conditions. The increase in systolic leaflet edge separation (average of the 4 time points during ejection) during $\mathrm{pLCx}$ coronary artery occlusion was $2.2 \pm 1.5 \mathrm{~mm}, 2.1 \pm 1.9 \mathrm{~mm}$, and $2.1 \pm 1.5 \mathrm{~cm}$ at the anterior commissure, valve center, and posterior commissure, respectively (all $P<.05$ ). No significant changes were observed during LAD coronary artery ischemia, and in only 2 instances did leaflet edge separation increase during $\mathrm{dLCx}$ coronary artery ischemia.

\section{Mitral Annular Dynamics}

Mitral annular area change during the cardiac cycle during each ischemic intervention is shown in Figure 4. Table 4 summarizes mitral annular area and S-L and C-C annular diameter measurements at end diastole during the 3 different ischemic interventions and the preischemic control conditions. The greatest amount of annular dilatation was observed during end diastole and beginning systole. Although significant annular dilatation ( $7 \% \pm 5 \%)$ was also present at end diastole during $\mathrm{dLCx}$ coronary artery ischemia, this was 
not associated with IMR. During pLCx coronary artery ischemia, however, mitral annulus area increased much more $(19 \% \pm 10 \%)$ and was associated with IMR. Similarly, the degree of mitral S-L diameter increase was much greater during proximal versus dLCx coronary artery occlusion $(18 \% \pm 9 \%$ vs $7 \% \pm 4 \%$ at end diastole, $P=.009)$. Interestingly, the IMR, which occurred during pLCx coronary artery occlusion, was not associated with any significant increase in the mitral annular $\mathrm{C}-\mathrm{C}$ dimension.

\section{Papillary Muscle Displacement, Geometry, and Systolic Shortening}

The distance in 3-D space between each papillary muscle tip and the midseptal annulus was calculated for each ischemic intervention to assess the effects of regional ischemia on LV geometry and the pathogenesis of IMR. The midseptal annulus was chosen as a reference site because it represents the center of the anterior fibrous annulus (which is relatively fixed and less influenced by the geometric perturbations induced by acute ischemia), is an identifiable echocardiographic landmark, and has been used previously in similar experimental studies. ${ }^{18}$ At end systole, LAD coronary artery occlusion was associated with $1.1 \pm 0.6 \mathrm{~mm}$ displacement of the posterior papillary tip away from the anterior mitral annulus $(P=.005$ vs preischemic control), and a similar displacement of $1.0 \pm 1.0 \mathrm{~mm}$ was seen during $\mathrm{dLCx}$ coronary artery occlusion $(P=.046$ vs preischemic control). During pLCx coronary artery ischemia, however, the displacement of the posterior muscle more than doubled $(2.6 \pm 0.8 \mathrm{~mm}, P=.0005)$, and there also was a $1.5 \pm 0.9$ $\mathrm{mm}(P=.003)$ displacement of the anterior papillary muscle away from the midseptal annulus. These changes throughout the cardiac cycle are shown in Figure 5. Thus significant IMR seen during pLCx coronary artery occlusion was associated with displacement of both papillary muscle tips away from the anterior annulus.

Papillary muscle shortening was calculated to assess whether papillary muscle dysfunction played a role in the pathogenesis of acute IMR. During LAD coronary artery occlusion, anterior papillary muscle shortening decreased from $15 \% \pm 4 \%$ to $3 \% \pm 4 \%(P=.003)$, but posterior papillary muscle shortening did not change $(16 \% \pm 7 \%$ vs $16 \% \pm 8 \%$ ). With $\mathrm{dLCx}$ coronary artery occlusion, there was no change in anterior papillary muscle shortening ( $14 \%$ $\pm 8 \%$ vs $14 \% \pm 9 \%$ ), but posterior papillary muscle shortening was markedly reduced $(16 \% \pm 8 \%$ vs $1.2 \% \pm 0.6 \%$, $P=.02)$, yet no IMR was seen. pLCx coronary artery occlusion produced a similar pattern of changes in papillary muscle shortening, as seen during $\mathrm{dLCx}$ coronary artery occlusion $(12 \% \pm 8 \%$ and $1 \% \pm 5 \%$ shortening for the anterior and posterior papillary muscles, respectively). These findings argue that isolated lack of systolic shortening of one or both papillary muscles does not affect mitral valve competence. The distance between the papillary muscle tips
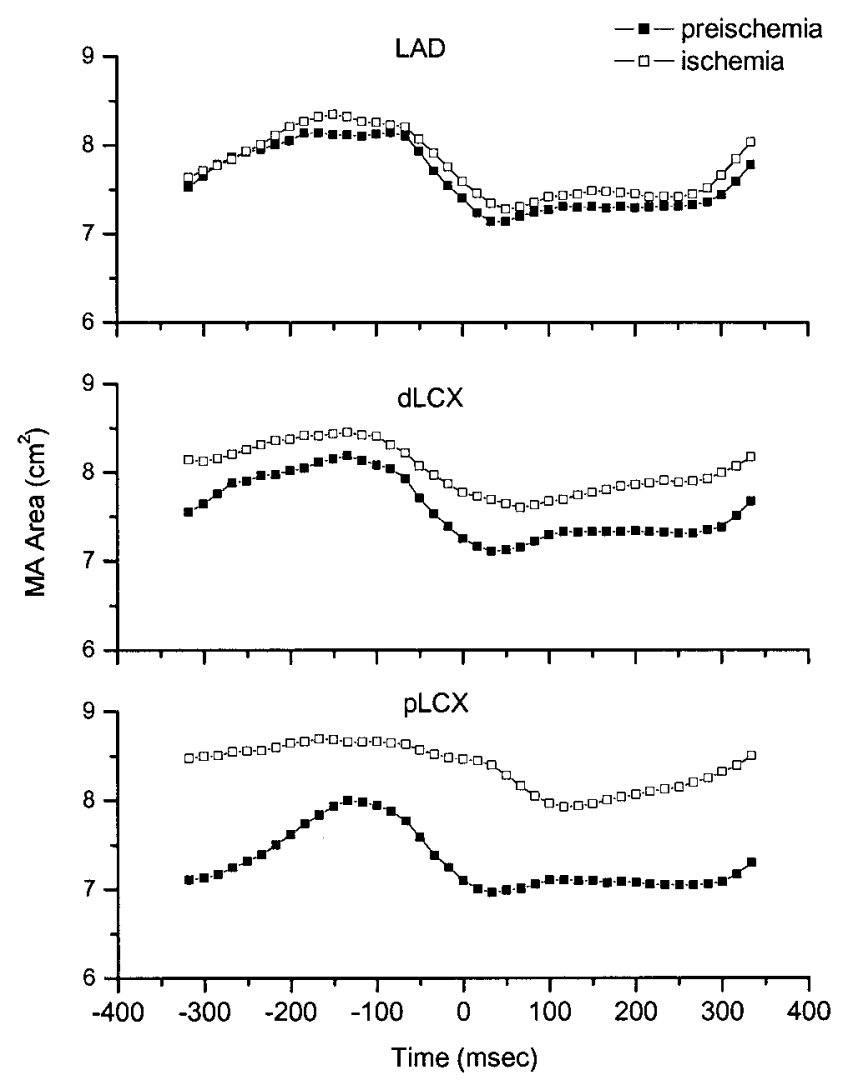

Figure 4. Average mitral annular (MA) area (in square centimeters) before (filled squares) and during (open circles) acute LV ischemia induced by balloon occlusion of either the LAD artery (top), the dLCx coronary artery (middle), or the pLCx coronary artery (bottom). A 650-ms time interval centered at end diastole $(t=0)$ is shown for all 3 groups.

at end-systole during LAD and $\mathrm{dLCx}$ coronary artery occlusion was larger than the respective preischemic control conditions (increase of $2.7 \pm 1.9 \mathrm{~mm}$ and $4.3 \pm 2.3 \mathrm{~mm}$ for LAD and $\mathrm{dLCx}$ coronary arteries, respectively; $P<.05$ ), but a much greater degree of papillary muscle tip separation occurred during pLCx coronary artery occlusion $(7.2 \pm 1.6$ $\mathrm{mm}, P=.0001$ ), suggesting greater perturbation of subvalvular 3-D geometry.

\section{Discussion}

IMR has been attributed to annular ${ }^{5,6}$ and subvalvular ${ }^{7-12}$ pathologic changes, altered leaflet geometry, ${ }^{10,14}$ and systolic dysfunction ${ }^{13}$ resulting from posterolateral ischemia, but these findings have not been evaluated in vivo during ischemia in other LV regions. The current study revealed that the extent of LV hemodynamic impairment did not directly cause acute IMR; only pLCx coronary artery occlusion resulted in acute IMR and not LAD or dLCx coronary artery occlusion. Analysis of 3-D marker measurements showed delayed early systolic valve closure and 

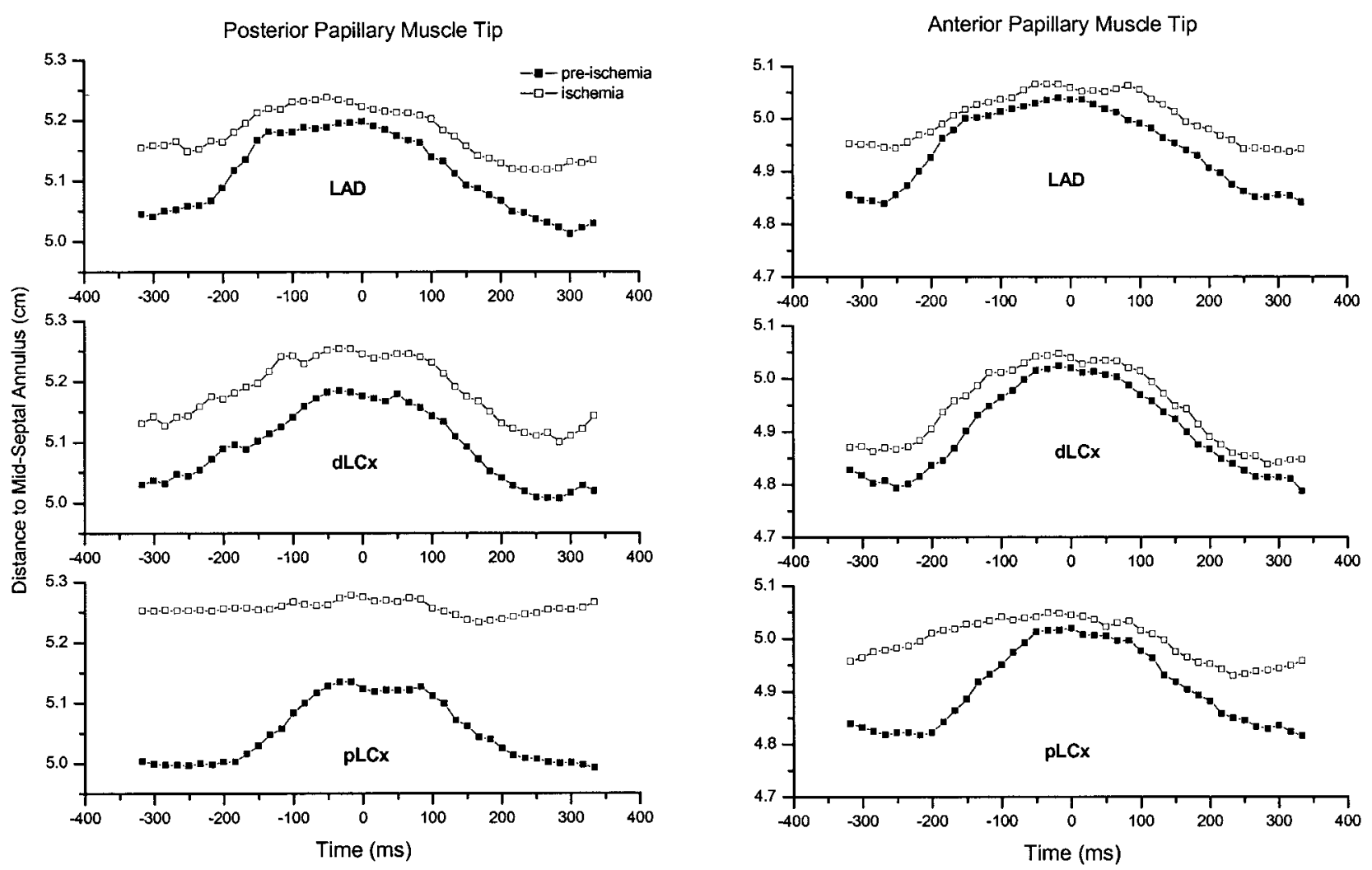

Figure 5. Group mean data for the distance from the posterior (left panel) and anterior (right panel) papillary muscle tips to the midseptal annulus before and during occlusion of each of the 3 coronary artery sites: LAD coronary artery (top), dLCx coronary artery (middle), or pLCx coronary artery (bottom). A 650-ms time interval centered at end diastole $(t=0)$ is shown for all graphs.

incomplete leaflet coaptation throughout systole across the entire valve. The IMR leak was predominantly central and was holosystolic.

We have previously reported that acute IMR in sheep was associated with delayed early systolic mitral valve closure. ${ }^{5}$ In the current experiment we observed this leaflet loitering in early systole at the center of the valve and also toward the commissures during pLCx coronary artery occlusion, which was accompanied by IMR. During LAD and dLCx coronary artery ischemia, delayed valve closure was not observed, and no significant IMR resulted. In addition to delayed early systolic mitral valve closure, incomplete mitral leaflet coaptation was seen throughout ejection during pLCx coronary artery ischemia. Leaflet edge separation throughout ejection increased by a similar extent at the valve center and at each commissure. Again, as with early systolic leaflet loitering, increased leaflet edge separation was only observed in conjunction with pLCx coronary artery occlusion but not during either LAD or dLCx coronary artery occlusion. Holosystolic leaflet edge separation was not observed in our previous study, ${ }^{5}$ probably because of a smaller ischemic insult and the fact that fewer leaflet markers were implanted. Therefore it appears that acute IMR in sheep is due to at least 2 underlying factors: delayed early systolic mitral valve closure and increased leaflet edge separation during the bulk of ejection.

The underlying geometric perturbations directly responsible for IMR are difficult to isolate. Previous ovine studies suggested that delayed early systolic valve closure was due to annular dilatation 5 ; in the current study, during $\mathrm{pLCx}$ coronary artery occlusion, annular dilatation was associated with IMR and leaflet loitering, whereas lesser degrees of annular dilatation during either LAD or $\mathrm{dLCx}$ coronary artery occlusion did not result in either IMR or delayed valve closure. This emphasizes the point that modest degrees of annular dilatation do not necessarily lead to acute IMR. ${ }^{19}$ The increase in the S-L annular diameter appeared to be key, whereas no dilatation was observed in the C-C distance. Thus increasing the annular dimension perpendicular to the line of leaflet coaptation (ie, S-L dilatation) probably plays a major role in delaying leaflet closure in early systole and increased leaflet separation subsequently throughout systole.

Restricted systolic leaflet motion caused by apical teth- 
ering or tenting, presumably from papillary muscle displacement, has also been observed in ovine acute IMR. ${ }^{14}$ Several investigators have postulated that perturbation of papillary muscle geometry prevents competent leaflet closure and results in IMR. ${ }^{6-8}$ The current analysis revealed that acute IMR requires displacement of both (but predominately the posterior) papillary muscle tips away from the septal annulus throughout ejection and at end systole, as was seen during pLCx coronary artery occlusion (Figure 5). Such papillary muscle displacement has been found in experimental ${ }^{8}$ and clinical ${ }^{6}$ studies to correlate with the presence of MR. In animal models of acute ${ }^{20}$ and chronic ${ }^{18}$ posterolateral ischemia, a larger distance from the papillary muscle tips to the midseptal annulus, measured by using 3-D echocardiography, was the only determinant of mitral regurgitant volume. Mitral annular dilatation was observed in both studies, being more prominent in the acute model. Indeed, during acute LCx coronary artery occlusion in dogs, displacement of the posteromedial papillary muscle tip away from the midseptal annulus was not sufficient to produce significant MR when the ventricle was kept small (intact pericardium and reduced LV preload), and mitral annular area remained unchanged. ${ }^{20}$ When the ventricle was allowed to dilate (open pericardium and increased LV volume) in this acute canine model, displacement of both (but particularly the ischemic posteromedial) papillary muscles tips away from the septal annulus was accompanied by approximately $22 \%$ annular dilatation and substantial MR. A similar degree of annular dilatation with papillary muscle displacement during IMR was observed in our experiment. Whether annular dilatation, papillary muscle displacement, or both is the mechanism primarily responsible for increased systolic leaflet separation cannot readily be distinguished in this model of acute IMR.

Decreased systolic shortening of either papillary muscle did not result in MR, which corroborates the findings of others $^{21}$ and is an additional demonstration that the old belief of papillary muscle dysfunction being responsible for IMR is invalid. Indeed, papillary muscle dysfunction has actually been demonstrated to ameliorate the severity of IMR. ${ }^{22}$ As suggested in the clinical literature, ${ }^{23,24}$ an additional insult to the LV wall underlying the papillary muscles is needed before valvular incompetence occurs. This notion is supported by the increased interpapillary distance and larger LV end-diastolic volume associated with IMR during pLCx coronary artery occlusion but not during dLCx coronary artery occlusion. This implies that a mild degree of LV chamber dilatation per se does not necessarily lead to IMR, as suggested by the very small and insignificant increase in LV end-diastolic volume seen with LAD occlusion $(P=$ .065); instead, the specific regional site and size of ischemic insult are actually more important. On the basis of ovine coronary artery anatomy and blood supply to the papillary muscles, ${ }^{25}$ one would expect that both proximal and $\mathrm{dLCx}$ coronary artery ischemia would result in posterior papillary muscle dysfunction, whereas LAD occlusion would affect anterior papillary muscle shortening. This was indeed observed in our study, yet IMR occurred only with $\mathrm{pLCx}$ coronary artery occlusion, probably because of the large area of underlying ischemic myocardium, which is associated with such an ischemic insult. ${ }^{25}$ IMR in this experiment was not associated with reduced $\mathrm{LV} \mathrm{dP} / \mathrm{dt}$, as previously suggested. ${ }^{13}$ Although a similar degree of LV systolic dysfunction was induced by the 3 ischemic interventions (Table 1 ), only pLCx coronary artery occlusion caused IMR. Others have also reported that the presence of IMR is not dependent on the degree of LV systolic dysfunction. 6,8 These data further support the hypothesis that regional alterations in valvular and subvalvular geometry play an important role in the pathogenesis of IMR.

There is a paucity of in vivo experimental data in the literature reporting the timing and location of IMR, but transesophageal echocardiography clinically is accurate in localizing the origin of the mitral regurgitant jets, ${ }^{26}$ including those of ischemic cause. ${ }^{27}$ Reports of in vitro studies of isolated porcine mitral valves are concordant with the echocardiographic and marker-derived findings reported herein. In an isolated in vitro beating heart preparation, $\mathrm{He}$ and colleagues $^{7}$ found that with annular dilatation and apicalposterolateral papillary muscle displacement, as can be seen in patients with IMR, MR occurred and was holosystolic, with prominent early and late systolic peaks. Using a similar experimental preparation, Nielsen and coworkers ${ }^{9}$ observed that displacement of both papillary muscles resulted in central MR. Annular dilatation as a consequence of previous myocardial infarction and LV dilatation in patients is commonly associated with central functional MR. ${ }^{28}$ Because both annular dilatation and displacement of both papillary muscle tips were observed in this acute experiment, the finding of holosystolic, central MR during pLCx coronary artery occlusion is consistent with these clinical observations. During pLCX coronary artery occlusion, leaflet separation distance was increased throughout systole at the valve center and also near each commissure, again demonstrating the occurrence of a broad central origin for the regurgitant jet. Not all patients with clinically significant IMR, however, have an echocardiographically demonstrated central regurgitant jet ${ }^{29}$; thus acute ovine IMR probably does not completely simulate the chronic IMR condition observed in human subjects.

The IMR during pLCx coronary artery occlusion in this experiment was accompanied by delayed early systolic valve closure and increased leaflet edge separation throughout ejection symmetrically around the valve center. Acute LV ischemic insults in other regions (ie, during LAD and dLCx coronary artery occlusion) did not lead to IMR, de- 
layed leaflet closure, or increased leaflet edge separation. We conjecture that annular dilatation was the key factor leading to delayed leaflet closure, whereas annular dilatation plus papillary muscle displacement during ejection caused increased leaflet edge separation. The individual contributions of these multiple mechanisms to the pathogenesis of IMR are difficult to isolate, but it is clear that the combination of valvular and ventricular geometric changes leads to central, holosystolic acute IMR in sheep.

Although this study overcomes some limitations of previous experiments, one must be circumspect in interpreting the results. These findings pertain only to the context of acute LV ischemia in the otherwise normal sheep hearts and should not be extrapolated to chronic human IMR, where LV dilatation, subvalvular geometric perturbations induced by previous infarction and ischemia, and LV remodeling most likely play a bigger role. This type of experiment needs to be conducted in chronic animal preparations after myocardial infarction with resultant LV remodeling and chronic LV volume overload to simulate more accurately the clinical problem. The present experiment, however, offers a unique opportunity to study the effects of annular and subvalvular geometric changes during 3 different regional LV ischemic insults in the same hearts, which cannot be done in a chronic model. Although the degree of systolic dysfunction induced by each ischemic intervention was similar, LV dP/dt (an isovolumic index) during pLCx coronary artery ischemia might have been artificially high because this was the only group with significant mitral insufficiency. Although this raises the possibility that the magnitude of the acute ischemic injury was greater during pLCx coronary artery occlusion, other indices of LV function decreased to a similar extent, suggesting equivalent hemodynamic insults.

The timing of IMR could be determined reliably in only 4 animals, and not all 6 animals had a central regurgitant jet; perhaps with more animals greater heterogeneity of regurgitant jets would have been observed. The differences in coronary anatomy between sheep and human subjects must also be considered because sheep left ventricles are more dependent on their dominant LCx coronary artery than human ventricles, in which right coronary artery dominance is most common. Several reliable models of human cardiac disease, however, have been established in ovine models. ${ }^{25,30}$ The radiopaque marker method provides reproducible determination of 3-D marker position with submillimeter spatial resolution every $16.7 \mathrm{~ms}$ but requires suturing small metal markers to the intracardiac structures. It is unlikely that the markers interfere with mitral leaflet motion because they are very small (aggregate mass, $20 \pm 6 \mathrm{mg}$ ); even when we grossly overloaded the anterior leaflet with a larger number of excessively heavy markers (total mass, $184 \mathrm{mg}$ ) in other animals, the peak anterior leaflet opening velocity by means of epicardial pulsed wave Doppler echocardiography was unchanged $(0.47 \pm 0.05 \mathrm{~m} / \mathrm{s}$ compared with $0.45 \pm 0.06 \mathrm{~m} / \mathrm{s}$ for leaflets without any markers implanted), as was the peak E-wave velocity ( 0.55 to 0.60 $\mathrm{m} / \mathrm{s}$, unpublished data).

We acknowledge the superb technical assistance provided by Carol W. Mead, BA, and Maggie Brophy, RVT.

\section{References}

1. Czer LSC, Gray RI, DeRobertis MA, Bateman TM, Stewart ME, Chaux A, et al. Mitral valve replacement: impact of coronary artery disease and determinants of prognosis after revascularization. Circulation. 1984;70(suppl I):I198-207.

2. Connolly MW, Gelbfish JS, Jacobowitz IJ, Rose DM, Mendelsohn A, Cappabianca PM, et al. Surgical results for mitral regurgitation from coronary artery disease. J Thorac Cardiovasc Surg. 1986;91:379-88.

3. Replogle RL, Campbell CD. Surgery for mitral regurgitation associated with ischemic heart disease. Circulation. 1989;79(suppl I): I122-5.

4. Dion R. Ischemic mitral regurgitation: when and how should it be corrected? J Heart Dis. 1993;2:536-43.

5. Glasson JR, Komeda M, Daughters GT, Bolger AF, Karlsson MO, Foppiano LE, et al. Early systolic mitral leaflet "loitering" during acute ischemic mitral regurgitation. J Thorac Cardiovasc Surg. 1998; 116:193-205.

6. Yiu SF, Enriquez-Sarano M, Tribouilloy C, Seward JB, Tajik AJ. Determinants of the degree of functional mitral regurgitation in patients with systolic left ventricular dysfunction. Circulation. 2000;102: 1400-6.

7. He S, Fontaine AA, Schwammenthal E, Yoganathan AP, Levine RA. Integrated mechanism for functional mitral regurgitation. Circulation. 1997;96:1826-34.

8. Otsuji Y, Handschumacher MD, Schwammenthal E, Jiang L, Song JK, Guerrero JL, et al. Insights from three-dimensional echocardiography into the mechanism of functional mitral regurgitation. Circulation. 1997;96:1999-2008.

9. Nielsen SL, Nygaard H, Fontaine AA, Hasenkam JH, He S, Yoganathan AP. Papillary muscle misalignment causes multiple mitral regurgitant jets: an ambiguous mechanism for functional mitral regurgitation. J Heart Valve Dis. 1999;8:551-64.

10. Gorman JH, Jackson BM, Gorman RC, Kelley ST, Gikakis N, Edmonds LH Jr. Papillary muscle discoordination rather than increased annular area facilitates mitral regurgitation after acute posterior myocardial infarction. Circulation. 1997;96(suppl II):II124-7.

11. Kono T, Sabbah HN, Rosman H, Alam M, Jafari S, Goldstein S. Left ventricular shape is the primary determinant of functional mitral regurgitation in heart failure. J Am Coll Cardiol. 1992;7:1594-8.

12. Sabbah HN, Kono T, Stein PD, Mancini GBJ, Goldstein S. Left ventricular shape changes during the course of evolving heart failure. Am J Physiol. 1992;263:H266-70.

13. Kaul S, Spotnitz WD, Glasheen PW, Touchstone DA. Mechanism of ischemic mitral regurgitation. Circulation. 1991;84:2167-80.

14. Lai DTM, Timek TA, Dagum P, Green GR, Glasson JR, Daughters GT, et al. The effect of ring annuloplasty on mitral leaflet geometry during acute left ventricular ischemia. J Thorac Cardiovasc Surg. 2000;120:966-75.

15. Glasson JR, Komeda M, Daughters GT, Foppiano LE, Bolger AF, Tye $\mathrm{TL}$, et al. Most ovine mitral annular three-dimensional size reduction occurs before ventricular systole and is abolished with ventricular pacing. Circulation. 1997;96(suppl II):II115-23.

16. Daughters GT, Sanders WJ, Miller DC, Schwarzkopf A, Mead CW, and Ingels NB. A comparison of two analytical systems for 3-D reconstruction from biplane videoradiograms. Comput Cardiol. 1988; 15:79-82.

17. Moon MR, DeAnda A, Daughters GT, Ingels NB, Miller DC. Experimental evaluation of different chordal preservation methods during mitral valve replacement. Ann Thorac Surg. 1994;58:931-44. 
18. Liel-Cohen N, Guerrero JL, Otsuji Y, Handschumacher MD, Rudski LG, Hunziker PR, et al. Design of a new surgical approach for ventricular remodeling to relieve ischemic mitral regurgitation: insight from 3-dimensional echocardiography. Circulation. 2000;101:275663.

19. Green GR, Dagum P, Glasson JR, Daughters GT, Bolger AF, Foppiano LE, et al. Mitral annular dilatation and papillary muscle dislocation without mitral regurgitation in sheep. Circulation. 1999; 100(Suppl II):II95-102.

20. Otsuji Y, Handschumacher MD, Liel-Cohn N, Tanabe H, Jiang L, Schwammenthal E, et al. Mechanism of ischemic mitral regurgitation with segmental left ventricular dysfunction: Three dimensional echocardiographic studies in models of acute and chronic progressive regurgitation. J Am Coll Cardiol. 2001;37:641-8.

21. Tsakiris AG, Rastelli GC, Amorim D, Titus JL, Wood EH. Effect of experimental papillary muscle damage on mitral valve closure in intact anesthetized dogs. Mayo Clin Proc. 1970;42:132-9.

22. Messas E, Guerrero JL, Handschumacher MD, Chow CM, Sullivan S, Schwammenthal E, et al. Paradoxic decrease in ischemic mitral regurgitation with papillary muscle dysfunction: insights form threedimensional and contrast echocardiography with strain rate measurement. Circulation. 2001;104:844-50.

23. Godley RW, Wann LS, Rogers EW, Feigenbaum H, Weyman AE. Incomplete leaflet closure in patients with papillary muscle dysfunction. Circulation. 1982;61:565-71.

24. Van Dantzig J, Delemarre BJ, Koster RW, Bot H, Visser CA. Pathogenesis of mitral regurgitation in acute myocardial infarction: importance of changes in left ventricular shape and regional function. Am Heart J. 1996;131:865-71.

25. Llaneras MR, Nance ML, Streicher JT, Lima JA, Savino JS, Bogen DK, et al. Large animal model of ischemic mitral regurgitation. Ann Thorac Surg. 1994;57:432-9.

26. Foster GP, Isselbacher EM, Rose GA, Torchiana DF, Akins CW, Picard MH. Accurate localization of mitral regurgitation defects using multiplane transesophageal echocardiography. Ann Thorac Surg. 1998;65:1025-31.

27. Enriquez-Sarano M, Freeman WK, Tribouilloy CM, Orszulak TA, Khandheria BK, Seward JB, et al. Functional anatomy of mitral regurgitation: accuracy and outcome implications of transesophageal echocardiography. J Am Coll Cardiol. 1999;34:1129-36.

28. Izumi S, Miyatake K, Beppu S, Park YD, Nagata S, Kinoshita N, et al. Mechanism of mitral regurgitation in patients with myocardial infarction: a study using real-time two-dimensional Doppler flow imaging and echocardiography. Circulation. 1987;76:777-85.

29. Gillinov AM, Wierup PN, Blackstone EH, Bishay ES, Cosgrove DM, White $\mathrm{J}$, et al. Is repair preferable to replacement for ischemic mitral regurgitation? J Thorac Cardiovasc Surg. 2001;122:1125-41.

30. Markovitz SE, Ratcliffe MB, Bavaria JE, Kreiner G, Iozzo RV, Hargrove WC, et al. Large animal model of left ventricular aneurysm. Ann Thorac Surg. 1989;48:838-45.

\section{Discussion}

Dr Henry M. Spotnitz (New York, NY). This is a very nice article and a very good use of the marker technique that was pioneered at Stanford. I would like to ask you a methodologic question. It is a bit unusual not to randomize a sequence of interventions. How did you arrive at that particular sequence of interventions, why didn't you randomize the order in which you did the various occlusions, and how do you know that your observations are not a function of the sequence in which you did this, especially because the appearance of many of the control tracings change over time and the controls clearly are not identical, particularly in the leaflet separation tracing that you showed?

Dr Timek. That is an excellent question. Our initial sequence of doing the occlusions really came from previous experiments. What we have noticed is that in sheep that undergo a $\mathrm{pLCx}$ coronary artery occlusion there is a very high frequency of ventricular fibrillation, and in a closed-chest preparation, once these animals go into ventricular fibrillation, the experiment is basically over. Therefore the way we proceeded was to induce the ischemic event, which is less likely to produce ventricular fibrillation first, that being LAD ischemia, dLCx, and then, at the end, pLCx.

Between each of these interventions, the animals were allowed to come to baseline as far as hemodynamics were concerned; however, you are correct, we cannot fully exclude that the sequence of the experiments somehow did not contribute to this. We are considering repeating this in an open-chest model in which ventricular fibrillation, when it does occur, can be treated much more easily.

Dr David H. Adams (New York, NY). I really enjoyed your talk. In the clinical setting ischemia in any circulatory territory can result in the phenomena of IMR, presumably on the basis of associated ventricular dilatation and papillary muscle displacement. Would you please comment on the mechanism of IMR in the setting of LAD occlusion or posterior descending artery occlusion, as opposed to pLCx occlusion?

Dr Timek. I think that one of the major limitations of this model is that this is acute IMR. We believe that some new data from our laboratory with chronic IMR, which has just been completed by my colleague, Dr Tibayan, shows that these changes in the chronic model are actually pretty similar to what we see in the acute model as far as pLCx coronary artery occlusion is concerned. We did not do any LAD ischemia in the chronic model, and that will be forthcoming.

As far as translating this into the clinical arena, the feeling is that clinical IMR is related to LCx ischemia and papillary muscle displacement. From our chronic and acute models, we believe that you really need both annular changes and you also need displacement of papillary muscles, and this really gets into how one should address IMR clinically. Is a ring sufficient, addressing basically the annular problem, or should there be some subvalvular interventions also? We have been working on some experimental surgical techniques to address these problems; however, this is still in its infancy.

Dr Adams. Just to follow up, I have one quick question about the research you just mentioned. Are you and Dr Miller's laboratory able to give us at least some insight into your comment? What geometric parameter might suggest that reduction annuloplasty is going to work, and would not further displacement or measurement of the papillary muscles suggest that we need to think about some papillary remodeling as well?

Dr Timek. If I may be allowed to give a prelude to my colleague's research, it is annular dilatation, at least in our models, which is really the key, particularly septal lateral dilatation. We presented an article last year just with isolated septal lateral reduction by a single suture that an acute model of IMR in sheep will abolish IMR. As far as the papillary muscle displacement is concerned, what we have found, surprisingly, is that there is displacement of the posterior papillary muscle, which is lateral and posterior but not apical. The muscle is not actually apically displaced, it is posterior and lateral, and in fact, it seems to move closer to the annular plane. There have been some suggestions of perhaps pulling the muscle up closer to the annulus. Whether that will work is difficult to say. 